\title{
Diagnosis and Treatment of Benign Prostatic Hyperplasia
}

\author{
Practice Patterns of Primary Care Physicians
}

Mary McNaughton Collins, MD, Michael J. Barry, MD, Lin Bin, PhD, Richard G. Roberts, MD, JD, Joseph E. Oesterling, MD, Floyd J. Fowler, PhD

OBJECTIVE: To define primary care physicians' (PCPs) practices in managing patients with benign prostatic hyperplasia (BPH), and to compare these practices to portions of the Agency for Health Care Policy and Research BPH guideline and urologists' practices.

DESIGN: Mail survey.

PARTICIPANTS: Nationwide random sample of PCPs and urologists, selected from the American Medical Association Registry.

METHODS: Initial mailing, postcard reminder, second mailing, telephone reminder, final mailing.

MAIN RESULTS: Primary care physicians $(n=444$, response $=$ $51 \%)$ reported seeing a median of 35 patients with BPH over the preceding year, in contrast to 240 for urologists ( $n=394$, response $=68 \%$ ). Regarding tests recommended by the guideline, two thirds of PCPs reported rarely or never using the American Urological Association (AUA) symptom index, nearly all reported routinely performing digital rectal examinations, and many (66\%) reported routinely ordering tests to determine the serum creatinine level. Although considered "optional" by the guideline, more than $\mathbf{9 0 \%}$ of PCPs reported routinely ordering a prostate-specific antigen test, while infrequently using other optional tests. Regarding "not recommended" studies, a substantial minority reported selectively or routinely ordering intravenous pyelography (34\%) and renal ultrasound (33\%), while two thirds reported rarely or never ordering these tests. Eighty-six percent of PCPs reported prescribing medications for BPH over the preceding year; $\alpha$ blockers to a median of 12 patients, and finasteride to a median of 2. Variation in urology referral thresholds was suggested in responses to two patient scenarios.

CONCLUSIONS: Primary care physicians are actively managing patients with BPH. Some of their diagnostic evaluations vary from the recommendations of a national guideline and urologists' practices. Referral thresholds appear to vary considerably.

KEY WORDS: prostatic hyperplasia; primary care physicians; practice patterns; practice guideline.

J GEN INTERN MED 1997;12:224-229.

$\mathbf{U}$ rologists have traditionally managed patients with benign prostatic hyperplasia (BPH), a common cause of morbidity among older men. ${ }^{1}$ Primary care physicians (PCPs) may now be playing a larger role in the care of $\mathrm{BPH}$ patients, in part owing to changes in the health care system that have altered the threshold for referrals. In addition, the Food and Drug Administration has approved several pharmacologic treatments for BPH (the $\alpha_{1}$-adrenergic blockers, terazosin and doxazosin, and the $5 \alpha$-reductase inhibitor, finasteride), and their availability may lead more PCPs to treat men with BPH themselves, with fewer referrals. ${ }^{2}$ Little is known, however, about whether PCPs are taking an active role in the management of $\mathrm{BPH}$ patients, and to what extent their practices conform to a clinical practice guideline, Benign Prostatic Hyperplasia: Diagnosis and Treatment, ${ }^{3}$ released in 1994 by the Agency for Health Care Policy and Research (AHCPR).

To explore the extent to which PCPs are involved in the diagnosis and treatment of $\mathrm{BPH}$, and to examine urology referral tendencies among them, we conducted a national survey of PCPs in 1995 focused on their management of BPH. We asked a nationwide sample of urologists similar questions for comparison. We also compared selfreported practices of PCPs to the recommendations of the AHCPR BPH guideline.

\section{METHODS}

\section{Sample Selection}

Using the American Medical Association Registry of Physicians, separate nationwide random samples were drawn of 450 listed general internists, 450 listed family physicians, and 600 listed urologists. We anticipated receiving responses from 300, 300, and 400 eligible physicians, respectively. With samples of this size, the 95\% confidence intervals around proportions for each sample would span only 5 to 6 percentage points at most. Comparisons between specialists and nonspecialists, when they were appropriate, would detect true differences of 6 percentage points (best case, if $P_{1}=.1$ or .9) to 9 percentage points (worst case, if $P_{1}=.5$ ) with $80 \%$ power. Physi-

Received from the General Medicine Division, Medical Services, Massachusetts General Hospital (MMC, MJB) and the Center for Survey Research, University of Massachusetts-Boston (LB, FJF), Boston; Department of Family Medicine, University of Wisconsin, Madison, (RGR); and Department of Urology, University of Michigan Medical Center, Ann Arbor (JEO).

Presented in part at the National meeting of the Society of General Internal Medicine, Washington, DC, May 4, 1996.

Supported by a PORT-II grant (HS 08397) from the Agency for Health Care Policy and Research. Dr. McNaughton Collins is supported by a General Medicine Research Fellowship (NRSA grant 5 T32 PE11001-08).

Address correspondence and reprint requests to Dr. Barry: Medical Practices Evaluation Center, Massachusetts General Hospital, 50 Staniford St., 9th Floor, Boston, MA 02114. 
cian offices were telephoned to verify that the potential participants' addresses and listings of specialty were correct, that they were practicing medicine at least 20 hours per week, and were not in residency training. Twenty-one general internists, 9 family physicians, and 14 urologists were ineligible, leaving a sample of 429 general internists, 441 family physicians, and 586 urologists.

\section{Survey Methods}

The mailing included a cover letter, a fact sheet describing the survey, a token \$5 payment, the survey instrument and a post-paid envelope. The initial mailing was followed by a postcard reminder and then another mailing of the survey instrument to nonrespondents. Subsequently, those physicians who had not yet responded after the third mailing were telephoned and encouraged to return the survey instrument. Physicians who had neither responded to date nor declined participation previously were mailed a final copy of the survey instrument.

\section{Survey Instrument}

Eligible physicians were mailed a pretested survey instrument, which took, on average, less than 20 minutes to complete. As part of the pretesting process, a separate group of primary care physicians and urologists completed earlier versions of the survey, and were debriefed in person or by telephone in order to improve the clarity and content of the questions. Two survey instruments were used for the study, one for primary care physicians and another for urologists. The instruments were designed to assess physicians' practice patterns regarding management of prostate diseases, both benign and malignant. The focus of this article is on the responses to those questions that pertain to the diagnosis and treatment of BPH by primary care providers. With respect to $\mathrm{BPH}$, categories of questions included diagnostic evaluation, treatment, and referral. Demographic and professional data from the physicians were also collected, including age, gender, percentage of time devoted to clinical practice, type of practice (solo vs group), and source of income derived from practice (salary vs fee for service).

The main survey question to primary care providers read: "When evaluating a male patient over 50 with symptoms of BPH to confirm a diagnosis, rule out other causes of urinary symptoms, or determine the severity of BPH before any urologic consultation, how often do you yourself use the following." A selective list of 10 tests followed with the response task: "Almost always," "More than half the time," "About half the time," "Less than half the time," "Rarely," and "Never." To simplify the presentation of our results, we collapsed the first two categories as "routine" use of a test, the next two as "selective" use, and the last two as "rarely or never" using a test.

The AHCPR guideline uses the terms "recommended," "optional," and "not recommended" to indicate degrees of desirability for routine use of specific diagnostic interventions. We asked about use of the AUA symptom index, serum creatinine level, and digital rectal examination, which are recommended; prostate-specific antigen (PSA), uroflowmetry, and postvoid residual, which are considered optional; and intravenous pyelography, renal ultrasound, and transrectal ultrasound, which are not recommended. Given our concerns about the length of the survey and the associated respondent burden, we did not include an exhaustive array of all examinations and tests addressed by the guideline in our survey. Regarding guideline-recommended tests, we did not include urinalysis or focused neurologic examination; regarding guidelineoptional tests, we did not include pressure-flow studies; and regarding tests not recommended by the guideline, we did not include filling cystometry or urethrocystoscopy.

The AUA symptom index, a 7-item self-administered questionnaire, is a simple, valid, and reliable way to quantify the severity of a patient's BPH symptoms, ${ }^{4,5}$ both for initial therapeutic decision making and for objectively measuring the response to therapy. According to the AHCPR guideline panel, the objective documentation of a patient's symptom level is the most essential part of its recommendations for the diagnosis, evaluation, treatment planning, and follow-up of patients with symptomatic BPH. ${ }^{3}$

\section{Statistical Analyses}

All analyses were performed on data from the completed survey instruments from the 444 eligible primary care physicians and 394 eligible urologists. Responses of family physicians and general internists were compared. For virtually all variables, no significant differences in distributions of responses between these two specialties could be detected; hence, for this article, the responses from the two groups of PCPs were combined. However, when there was a difference in response distributions by primary care specialty, that difference is noted in the relevant table.

When primary care physicians and urologists answered identical questions, the distributions of responses were compared using the $\chi^{2}$ statistic or Fisher's Exact Test when appropriate. This analytic approach does not assume that the urologists have the right answers; rather, the practices of urologists provide a context within which to interpret the answers from primary care physicians. The urologists' practices have been reported previously. ${ }^{6}$

Descriptive analyses were completed using the Statistical Package for the Social Sciences. Continuous responses (such as numbers of patients seen or prescriptions written) were not normally distributed and therefore were summarized by median and interquartile range (IQR). The IQRs represent the 25 th and 75 th percentiles of the distributions. Comparisons of responses by physician or practice characteristics were assessed for statistical significance using the $\chi^{2}$ statistic or the Wilcoxon Rank-Sum Test, depending on whether the examined variables were categorical or continuous, respectively. ${ }^{7}$ 


\section{RESULTS}

\section{Study Population}

Fifty-one percent of sampled primary care physicians ( $n=870$ ) responded, yielding a total of 444 completed surveys (218 from general internists, 226 from family physicians). Sixty-eight percent of sampled urologists ( $n=583$ ) responded, yielding a total of 394 completed surveys. Table 1 presents some characteristics of the responding physicians in the sample. Primary care physicians were, on average, younger, more often female, and more frequently salaried than urologists. Physicians were asked how many different patients with a diagnosis of BPH they had seen over the past 12 months. Primary care physicians reported seeing a median of 35 (IGR 20-87) different BPH patients over the past year, and urologists reported seeing a median of 240 (IQR 110-500).

\section{Diagnosis of Benign Prostatic Hyperplasia}

Physicians were asked how they would initially evaluate a man over age 50 with symptoms suggesting BPH. Table 2 displays the PCPs' and urologists' reported frequencies of performing various examinations and tests, and includes many of the AHCPR BPH guideline recommendations.

Regarding guideline-recommended diagnostic examinations and tests, almost two thirds of PCPs reported that they rarely or never used the AUA symptom index, while two thirds of urologists reported routinely using this instrument. In contrast, two thirds of PCPs, but only about one quarter of urologists, reported routinely ordering tests for serum creatinine levels. Nearly all PCPs and all urologists reported performing digital rectal examinations routinely.

Although the AHCPR BPH guideline considers PSA testing optional, more than 90\% of PCPs and 95\% of urologists reported routinely ordering a PSA test. Primary care physicians reported a very low frequency of ordering other guideline-optional tests; $95 \%$ reported rarely or never ordering uroflowmetry, and $79 \%$ and $88 \%$ reported a similar frequency of ordering postvoid residual volume by catheterization and by ultrasound, respectively. Urologists, in contrast, tended to perform these tests more frequently, particularly uroflowmetry and postvoid residual volume by ultrasound.

Regarding the upper tract imaging studies, which are not recommended by the guideline, a substantial minority of PCPs reported selectively or routinely ordering intravenous pyelography $(34 \%)$ or renal ultrasound (33\%), while two thirds reported rarely or never ordering these two tests; $83 \%$ reported rarely or never ordering transrectal ultrasound. Neither group ordered ultrasounds very often, although urologists selectively performed these tests more frequently than PCPs.

\section{Predictors of Primary Care Physician Responses}

In general, primary care physicians' characteristics (including the volume of $\mathrm{BPH}$ patients seen) were not sig-
Table 1. Background Characteristics of the Primary Care Physician and Urologist Samples*

\begin{tabular}{lccc}
\hline \hline Characteristic & $\begin{array}{c}\text { PCPs } \\
(\boldsymbol{n}=\mathbf{4 4 4 )}\end{array}$ & $\begin{array}{c}\text { Urologists } \\
(\boldsymbol{n}=\mathbf{3 9 4})\end{array}$ & $\boldsymbol{p}$ Value \\
\hline \% Clinical time & & & \\
$\quad<90 \%$ & 12 & 12 & $=.99$ \\
$\quad>90 \%$ & 88 & 88 & \\
Type of practice, ${ }^{\dagger} \%$ & & & \\
$\quad$ Solo & 35 & 31 & $<.05$ \\
$\quad$ Single-specialty group & 42 & 53 & \\
$\quad$ Multispecialty group & 23 & 16 & \\
Income type, \% & & & \\
$\quad$ Salaried & 31 & 12 & $<.0001$ \\
$\quad$ Mixed & 20 & 12 & \\
Fee for service & 49 & 76 & \\
Age & & & \\
$\quad<40$ years & & 18 & \\
40-49 years & 29 & 30 & $<.0001$ \\
50-59 years & 35 & 34 & \\
$\geq 60$ years & 17 & 18 & \\
Gender, \% & 19 & & \\
Male & & 99 & $<.0001$ \\
Female & 84 & 1 & \\
\hline
\end{tabular}

*PCP indicates primary care physician.

${ }^{\dagger}$ For type of practice and age, there were differences between PCP groups. General internists were older $(\mathrm{p}=.012)$ and more often in solo practice $(\mathrm{p}=.0001)$ than family physicians.

nificantly associated with the likelihood that they would perform various diagnostic examinations and tests for BPH. Some exceptions were that primary care physicians who characterized their primary medical practice as solo reported ordering intravenous pyelograms (a test not recommended by the AHCPR guideline) more often than those who characterized their primary medical practice as a single-specialty group or multispecialty group (11\% vs $4 \% ; p=.006)$. Also, physicians in exclusively fee-for-service or mixed practice arrangements reported ordering intravenous pyelograms more often than physicians who were completely salaried ( $38 \%$ vs $24 \% ; p=.005$ ). Although older primary care physicians reported ordering a serum creatinine test (recommended by the guideline) more often than younger physicians $(74 \%$ vs $61 \%$; $p=$ .007), they also reported ordering intravenous pyelograms more often (70\% vs $54 \% ; p=.001)$.

\section{Medical Management}

Asked whether they had prescribed any medications to treat BPH over the past 12 months, $86 \%$ of PCPs answered yes. Asked how they had initiated medical therapy in the past 12 months, PCPs reported prescribing $\alpha$ blockers more often than finasteride. PCPs newly prescribed $\alpha$ blockers to a median of 12 (IQR 4-25) BPH patients over the past 12 months, and finasteride to a median of 2 (IQR 0-6) BPH patients. Urologists similarly initiated prescriptions for $\alpha$-blockers (median 70; IQR 30-125) more often than finasteride (median 15; IQR 5-40). 
Table 2. Reported Frequency of Performing Agency for Health Care Policy and Research Guideline Examinations and Tests for Benign Prostatic Hyperplasia by Primary Care Physicians $(n=444)$ and Urologists $(n=394)^{*}$

\begin{tabular}{|c|c|c|c|c|c|c|}
\hline AHCPR Guideline & Test & Physician Type & $\%$ Routine & $\%$ Selective & $\%$ Rarely/Never & $p$ Value \\
\hline & AUA symptom index & Urologists & 63 & 18 & 18 & .001 \\
\hline & Creatinine level $^{\dagger}$ & Urologists & 26 & 34 & 39 & .001 \\
\hline & \multirow[t]{2}{*}{ Digital rectal exam } & PCPs & 98 & 1 & 1 & \multirow[t]{2}{*}{.011} \\
\hline & & Urologists & 100 & 0 & 0 & \\
\hline & PSA test ${ }^{\ddagger}$ & Urologists & 95 & 4 & 1 & .049 \\
\hline & \multirow[t]{2}{*}{ Uroflowmetry } & PCPs & 1 & 4 & 95 & \multirow[t]{2}{*}{.001} \\
\hline & & Urologists & 40 & 28 & 32 & \\
\hline & \multirow[t]{2}{*}{ Postvoid residual volume, catheter $\ddagger$} & PCPs & 4 & 17 & 79 & \multirow[t]{2}{*}{.001} \\
\hline & & Urologists & 14 & 33 & 54 & \\
\hline & Postvoid residual volume, ultrasound & PCPs & 3 & 9 & 88 & .001 \\
\hline & \multirow[t]{2}{*}{ Renal ultrasound\$ } & PCPs & 7 & 26 & 67 & \multirow[t]{2}{*}{.001} \\
\hline & & Urologists & 8 & 38 & 54 & \\
\hline & \multirow[t]{2}{*}{ Transrectal ultrasound } & PCPs & 4 & 13 & 83 & \multirow[t]{2}{*}{.001} \\
\hline & & Urologists & 5 & 31 & 64 & \\
\hline
\end{tabular}

* More than 95\% of respondents answered each question. AHCPR indicates Agency for Health Care Policy and Research; AUA, American Urological Association; PCP, primary care physician; PSA, prostate-specific antigen.

${ }^{\dagger}$ For creatinine tests there were significant differences between PCP groups. More general internists than family physicians reported routinely ordering creatinine tests $(\mathrm{p}<.0001)$.

${ }^{\ddagger}$ For PSA and postvoid residual volume by catheter, there were significant differences between PCP groups. More general internists than family physicians reported routinely ordering PSA $(\mathrm{p}=.0002)$. More family physicians than general internists reported ordering postvoid residual volume by catheterization $(\mathrm{p}=.0124)$.

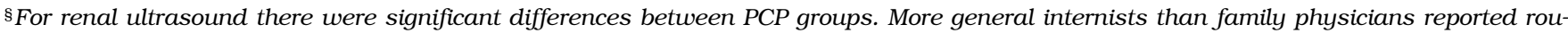
tinely ordering renal ultrasound $(\mathrm{p}=.0045)$.

\section{Specialty Referrals}

When asked how many patients they had referred to a urologist specifically for suspected or confirmed $\mathrm{BPH}$ over the past 12 months, PCPs reported a median of 10 (IQR 4-20) referrals. As noted above, PCPs reported seeing a median of 35 patients with a diagnosis of BPH over the same interval.

Next, PCPs were given two patient scenarios-scenarios describing patients that, according to the AHCPR guideline, would be reasonable for a PCP to manage without a urology referral. Table 3 provides both scenarios and the PCPs' responses regarding referral in these situations. In the first case, a patient with moderate urinary symptoms consistent with BPH but no findings suggestive of cancer, more than one third of the PCPs reported that they would refer to a urologist about half the time or more, while another $40 \%$ reported that they would rarely or never refer such a patient. In the second case, a patient with a larger than average prostate on digital rectal examination but no urinary symptoms and no findings suggestive of cancer, almost one fourth of PCPs reported they would refer to a urologist about half the time or more, while another $60 \%$ would rarely or never refer such a pa- tient. Primary care physicians who reported seeing fewer than the median of 35 patients with BPH over the past year more often answered that they would consult a urologist routinely in response to the two case scenarios, compared with PCPs who saw more than the median (case $1,29 \%$ vs $15 \%, p=.001$; case $2,22 \%$ vs $11 \%, p=.001$ ).

\section{DISCUSSION}

We found that PCPs are actively managing patients with $\mathrm{BPH}$. When prescribing medications for $\mathrm{BPH}$, they reported choosing $\alpha$ blocker therapy more often than finasteride; this finding predated a recent controlled trial documenting the superior effectiveness of terazosin compared with finasteride. ${ }^{8}$ The PCPs' diagnostic evaluations tended to vary from some of the recommendations of the AHCPR BPH guideline, as well as from some of the practices of their urologic colleagues. The urologists' diagnostic evaluations, however, also sometimes differed from the guidelines. In addition, from their responses to the two case scenarios, our findings suggest that there is considerable variation among primary care physicians in referral thresholds. Finally, the volume of BPH patients seen by 
Table 3. Responses Regarding Primary Care Physicians' Referrals to Urology in Two Different Clinical Scenarios, $(n=444)$

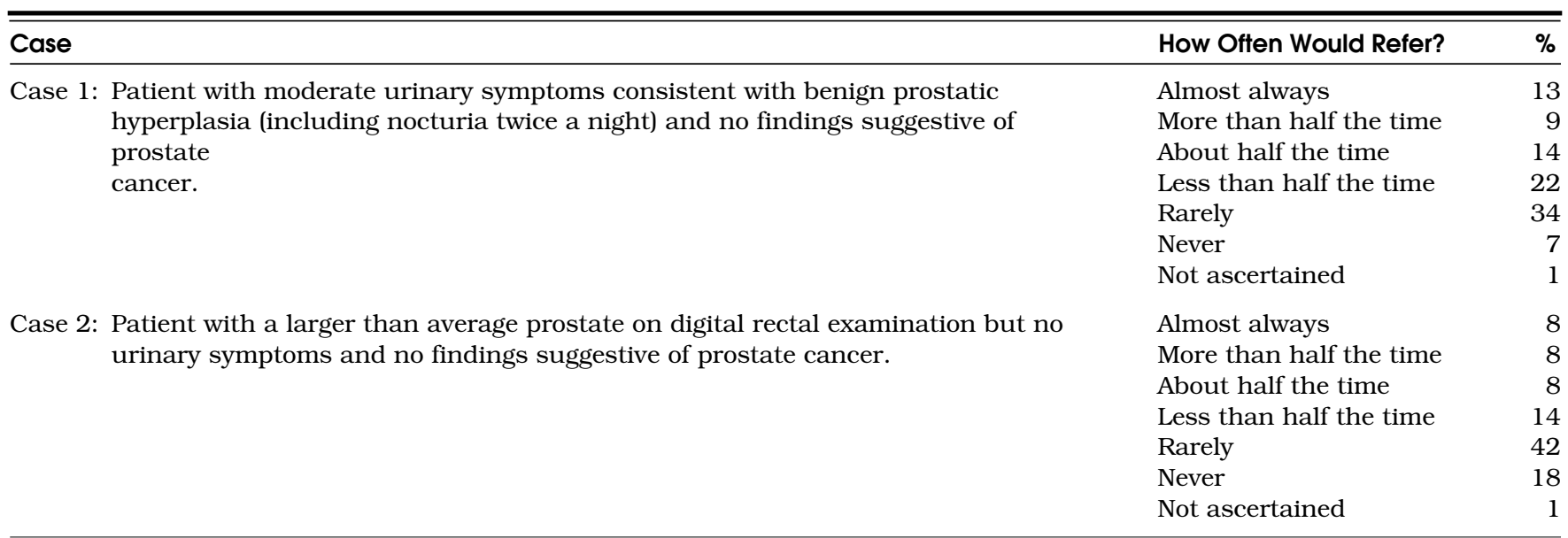

PCPs seemed to influence their reported referral thresholds; those who saw fewer patients were more likely to refer in both cases.

The responses concerning some diagnostic tests (especially the use of the AUA symptom index, PSA tests, and upper tract imaging studies) suggest that some PCPs are either unaware of or noncompliant with recommendations from the AHCPR BPH guideline for a patient-centered, evidence-based, and cost-effective evaluation of suspected $\mathrm{BPH}$. For example, many PCPs reported rarely or never using the guideline-recommended AUA symptom index, which provides a reliable and valid way to measure a patient's symptom severity. Our results parallel those of a regional survey of Brooklyn PCPs by Plawker and colleagues, ${ }^{9}$ which found only $33.5 \%$ of responding PCPs were aware of the AUA symptom index. In contrast, the urologists that we surveyed commonly used this instrument; 45\% reported "almost always" using the AUA score, and another 19\% reported using it "more than half the time." Also, given that the vast majority of PCPs acknowledged prescribing medications to treat BPH, use of the AUA symptom index could present an opportunity for more objectively monitoring response to therapy. In the Plawker study, only $15 \%$ of PCPs prescribing pharmacotherapy for $\mathrm{BPH}$ reported administering the AUA symptom index. ${ }^{9}$ An editorial accompanying the Plawker article, written by a urologist, implores urologists to educate their "nonurologic colleagues"-who are described as having "important misconceptions and deficiencies" regarding male voiding dysfunction. ${ }^{10}$

Both the PCPs and urologists in our study almost unanimously reported ordering PSA tests in the evaluation of suspected $\mathrm{BPH}$, a practice deemed optional by the AHCPR BPH guideline. According to Meigs and colleagues, ${ }^{11}$ the ability of the PSA test to discriminate men with localized prostate cancer from men without prostate cancer is considerably reduced in the setting of $\mathrm{BPH}$, so the PSA testing appears to make less sense in men with $\mathrm{BPH}$ than in unselected men. In contrast to previous beliefs, lower urinary tract symptoms suggestive of $\mathrm{BPH}$ do not appear to increase the risk that a patient harbors a localized prostate cancer that may be discovered by PSA testing. ${ }^{12,13}$ In addition, many BPH patients are aged 75 years or older (suggesting a less than 10-year life expectancy), ages when the value of early detection and treatment of prostate cancer is in doubt, even by advocates of PSA testing. ${ }^{14,15}$ A relatively high proportion of both PCPs and urologists also reported ordering upper tract imaging studies in their initial BPH evaluation-an expensive practice of marginal value (and small but finite risk in the case of intravenous pyelography), which is not recommended by the AHCPR BPH guideline.

There appeared to be considerable variation among PCPs in their thresholds for urologic referrals, as reflected in responses to questions about the two patient scenarios. As the health care system is currently in the midst of rapid changes, with primary care physicians being asked to assume a larger role in the care of patients who in the past may have been routinely referred to specialists, a certain amount of practice variation is to be expected. With time, PCPs may become more comfortable caring for patients with many common diseases that only a short time ago they would have referred to specialists; urologic conditions, such as BPH, may be a prime example. However, dissemination of information on BPH management to PCPs is crucial. ${ }^{16}$ Publications on the management of BPH used to appear mainly in journals that targeted urologists, but more recently the information on $\mathrm{BPH}$ diagnosis and treatment is appearing in journals with a general readership. ${ }^{17-20}$

Our findings must be interpreted with caution because the data on the practice patterns of PCPs were based on physician self-report, and the PCP response rate was relatively low (51\%). Although nonresponse may have biased these results, PCPs less knowledgeable and inter- 
ested in prostate diseases are more likely to have been among the nonresponders. Thus, our results indicating a potential lack of awareness of or noncompliance with the AHCPR BPH guideline may be understated. Unfortunately, we have no demographic information on the nonrespondents to allow a comparison with our respondents. Also, the associations between the physician characteristics and management practices must be viewed with caution, given that multiple tests of association were performed. Another potential limitation to our study is that given concerns about respondent burden, we did not exhaustively ask about every historical point physicians might ask about in response to a patient with lower urinary tract symptoms suggesting $\mathrm{BPH}$.

The rapid diffusion of managed care has changed how referrals to specialists are made, generating controversy over what aspects of care should be provided by generalists versus specialists. ${ }^{21-23}$ The development and dissemination of information in the form of clinical practice guidelines, though controversial, ${ }^{24-26}$ is one remedy commonly suggested for improving care provided by generalists. Multifaceted interventions appear most successful in changing physician practices. ${ }^{27}$ Both the evolution of the health care system toward a greater focus on primary care and the availability of nonsurgical treatments for $\mathrm{BPH}$ are moving more and more $\mathrm{BPH}$ management under the direction of PCPs. Increased awareness of and compliance with the AHCPR BPH clinical practice guideline may facilitate PCPs provision of more effective diagnosis and treatment for their patients with BPH.

The authors thank all the participating primary care physicians and urologists for making this study possible.

\section{REFERENCES}

1. Barry MJ, Boyle P, Fourcroy J, et al. Epidemiology and natural history of BPH. In: Cockett ATK, Khoury S, Aso Y, et al. The 3rd International Consultation on BPH: Proceedings. Paris, France: Scientific Communication International; 1996:21-50.

2. Monda JM, Oesterling JE. Medical treatment of benign prostatic hyperplasia: 5-alpha-reductase inhibitors and alpha-adrenergic antagonists. Mayo Clin Proc. 1993;68:670-9.

3. McConnell JD, Barry MJ, Bruskewitz RC, et al. Benign prostatic hyperplasia: Diagnosis and Treatment. Clinical Practice Guideline, No 8. Rockville, Md: Agency for Health Care Policy and Research, Public Health Service, United States Department of Health and Human Services; 1994. AHCPR, Publication No. 94-0582.

4. Barry MJ, Fowler FJ, O'Leary MP, et al. The American Urological Association symptom index for benign prostatic hyperplasia. J Urol 1992;148:1549-57.

5. O'Leary MP, Barry MJ, Fowler FJ. Hard measures of subjective out- comes: validating symptom indexes in urology. J Urol 1992;148: 1546-8.

6. Barry MJ, Fowler FJ, Bin L, Oesterling JE. A nationwide survey of practicing urologists: current management of benign prostatic hyperplasia and clinically localized prostate cancer. J Urol. In press.

7. Rosner B. Fundamentals of Biostatistics. Boston, Mass: PWSKent; 1990.

8. Lepor $\mathrm{H}$, Williford WO, Barry MJ, et al. The efficacy of terazosin, finasteride, or both in benign prostatic hyperplasia. N Engl J Med. 1996;335:533-9.

9. Plawker MW, Fleisher JM, Nitti VW, Macchia RJ. Primary care practitioners: an analysis of their perceptions of voiding dysfunction and prostate cancer. J Urol. 1996;155:601-4.

10. Partin AW. Prostate cancer-educating ourselves and our nonurological colleagues. J Urol. 1996;155:610-11. Editorial.

11. Meigs JB, Barry MJ, Oesterling JE, Jacobsen SJ. Does prostatespecific antigen testing make less sense for men with prostatic hyperplasia than for unselected men? J Gen Intern Med. 1996;11: 505-12.

12. Barry MJ, Fleming C, Coley CM, Wasson JH, Fahs MC, Oesterling JE. Should Medicare provide reimbursement for prostate-specific antigen testing for early detection of prostate cancer? Part I: framing the debate. Urology. 1995;46:2-13.

13. Catalona WJ, Richie JP, Ahman FR, et al. Comparison of digital rectal examination and serum prostate specific antigen in the early detection of prostate cancer: results of a multicenter clinical trial of 6,630 men. J Urol. 1994;151:1283-90.

14. Walsh PC. Using prostate-specific antigen to diagnose prostate cancer: sailing in uncharted waters. Ann Intern Med. 1993;119: 948-9.

15. Middleton RG, Thompson IM, Austenfeld MS, et al. Prostate cancer clinical guidelines panel summary report on the management of clinically localized prostate cancer. J Urol. 1995;154:2144-8.

16. Stross JK, Harlan WR. The dissemination of new medical information. JAMA. 1979;241:2622-4.

17. Jacobsen SJ, Girman CJ, Guess HA, et al. New diagnostic and treatment guidelines for benign prostatic hyperplasia: potential impact in the United States. Arch Intern Med. 1995; 155:477-81.

18. Roberts RG. BPH: new guidelines based on symptoms and patient preference. Geriatrics. 1994;49:24-31.

19. Oesterling JE. Benign prostatic hyperplasia: medical and minimally invasive treatment options. N Engl J Med. 1995;332:99-109.

20. McNaughton Collins MF, Friedman RH, Ash A, Hall R, Moskowitz MA. Underdetection of clinical benign prostatic hyperplasia in a general medicine practice. J Gen Intern Med. 1996;11:513-8.

21. Kassirer JP. Access to specialty care. N Engl J Med. 1994;331: 1151-3. Editorial.

22. Greenfield S. Dividing up the turf: generalists versus subspecialists. J Gen Intern Med. 1996;11:245-6. Editorial.

23. Neilson KG. When does a generalist need a specialist? J Gen Intern Med. 1996;11:247-8. Editorial.

24. Eddy DM. Clinical decision making: from theory to practice; three battles to watch in the 1990's. JAMA. 1993;270:520-6.

25. Lee TH. Beyond guidelines: can general internists show the (critical) paths? J Gen Intern Med. 1996;11:174-5.

26. Hayward RSA, Wilson MC, Tunis SR, Guyatt GH, Moore KA, Bass EB. Practice guidelines: what are internists looking for? J Gen Intern Med. 1996;11:176-8.

27. Greco PJ, Eisenberg JM. Changing physicians' practices. N Engl J Med. 1993;329:1271-4. 